\title{
Cyclotron resonance and electron-electron interactions in the organic metal (BEDT-TTF) ${ }_{2} \mathrm{KHg}(\mathrm{SCN})_{4}$
}

\author{
M. Doporto ${ }^{a}$, J. Singleton ${ }^{a}$, F.L. Pratt ${ }^{a}$, T.J.B.M. Janssen ${ }^{b}$, J.A.A.J. Perenboom ${ }^{\text {, }}$ \\ M. Kurmoo', W. Hayes ${ }^{a}$ and P. Day ${ }^{\text {c }}$ \\ ${ }^{a}$ Clarendon Laboratory, Department of Physics, University of Oxford, Parks Road, Oxford OX1 $3 P U, U K$ \\ ${ }^{\mathrm{b}}$ High Field Magnet Laboratory, NL-6525 ED Nijmegen, The Netherlands \\ 'Inorganic Chemistry Laboratory, South Parks Road, Oxford, UK \\ ${ }^{d}$ Institut Laue Langevin, BP 156X, Avenue des Martyrs, 38042 Grenoble Cedex, France
}

\begin{abstract}
We report the first cyclotron resonance measurements on (BEDT-TTF $)_{2} \mathrm{KHg}(\mathrm{SCN})_{4}$, which yield an effective mass of $m_{\text {cyc }}^{*}=(0.40 \pm 0.02) m_{\mathrm{e}}$ at $1.2 \mathrm{~K}$; transport measurements give $m_{\mathrm{tr}}^{*}=2.0 m_{\mathrm{e}}$. Using Kohn's theorem we propose that the enhancement of $m_{\mathrm{tr}}^{*}$ over $m_{\mathrm{cyc}}^{*}$ is the first direct gauge of the electron-electron interaction in a metallic charge transfer salt.
\end{abstract}

Charge-transfer salts of the ET molecule [1] form a versatile system for the study of band formation; by varying the anion $X$ in the salt $(\mathrm{ET})_{n} \mathrm{X}$, the stoichiometry and band-filling may be adjusted, producing metallic semimetallic, or semiconducting compounds with quasi-twodimensional (Q2D) electronic properties, some of which may also be superconductors [2]. The compounds are also of interest in the study of electron-electron interactions (EEI) in narrowbandwidth metallic systems; e.g. we have noted that there is a large enhancement of the transport effective mass over the predicted band mass in metallic $\mathrm{ET}_{2} \mathrm{Cu}(\mathrm{SCN})_{2}$ [3], thought to be due to electron-electron interactions $[3,4]$.

Experimental techniques which gauge the Fermi surface (FS) are making a great contribution to the understanding of these materials. Most studies have involved measurements of the Shubnikov-de Haas oscillations ( $\mathrm{SdHo}$ ) in the magnetoresistance (MR) [5]; there have also been a few de Haas-van Alphen studies [6]. In contrast, no cyclotron resonance (CR) studies have been reported (although an apparent CR has recently been observed in the field-inducedspin-density-wave state of $\mathrm{TMTSF}_{2} \mathrm{ClO}_{4}$ [7]). $\mathrm{CR}$ measurements have an important role in the study of EEI since, according to Kohn's theorem
[8], the effective mass measured by $\mathrm{CR}$ is independent of EEI, giving the band mass renormalised only by electron-phonon interactions, whereas the transport mass, measured e.g. by $\mathrm{SdHo}$, will additionally be influenced by the EEI effects. In this paper we report the first far-infrared (FIR) optical measurements of the cyclotron mass of the closed section of the Fermi surface in metallic $\mathrm{ET}_{2} \mathrm{KHg}(\mathrm{SNC})_{4}$. In addition, we have measured the EEI renormalised mass using $\mathrm{SdHo}$, which is found to be several times larger than the cyclotron mass, providing clear evidence of the importance of EEI in this material.

Measurements were performed on crystals of $\mathrm{ET}_{2} \mathrm{KHg}(\mathrm{SCN})_{4}$ grown electrochemically using ET prepared by the method of Larsen and Lenoir [9], and KSCN, HgSCN and 18Crown6 in a mixture of $90 \%, 1,1,2-\mathrm{TCE}$ and $10 \%$ ethanol. Crystals formed as small black distorteddiamond shaped platelets. This salt has the typical layer structure for an ET salt resulting in highly conducting planes separated by layers of anions. The ET molecules are arranged in a herringbone pattern with the layers.

$\mathrm{ET}_{2} \mathrm{KHg}(\mathrm{SCN})_{4}$ is metallic down to at least $400 \mathrm{mK}$ and is known to be in an antiferromagnetic ground state below $\sim 10 \mathrm{~K}$. The salt has 
attracted much attention recently due to the spin-split structure of the SdHo and the anomalous high-field kink at $\sim 23 \mathrm{~T}$ in the $\mathrm{MR}$ seen in early studies [10]; our MR measurements on this material [11] have shown a number of new features. Above $23 \mathrm{~T}$, the SdHo, which show only one frequency (plus harmonics) at $\sim 670 \mathrm{~T}$, and the angle-dependent MR are both in fair agreement with the theoretical FS predicted using the extended Hückel model [12], which has only one closed orbit (see inset to fig. 1). Below the kink feature, however, there is also a sample-dependent higher frequency in the SdHo which can be as large as $\sim 800 \mathrm{~T}$ [11]. The extra frequency is thought to result from the effect of inter-plane antiferromagnetic ordering on the band structure and thus the kink feature may result from a field-induced switching of the inter-plane ordering from antiferromagnetic to ferromagnetic [11].

For the CR measurements the crystals were arranged in the form of a mosaic with the highly conducting planes perpendicular to the magnetic field. The FIR laser system used has been described in detail elsewhere [13]; two carbon

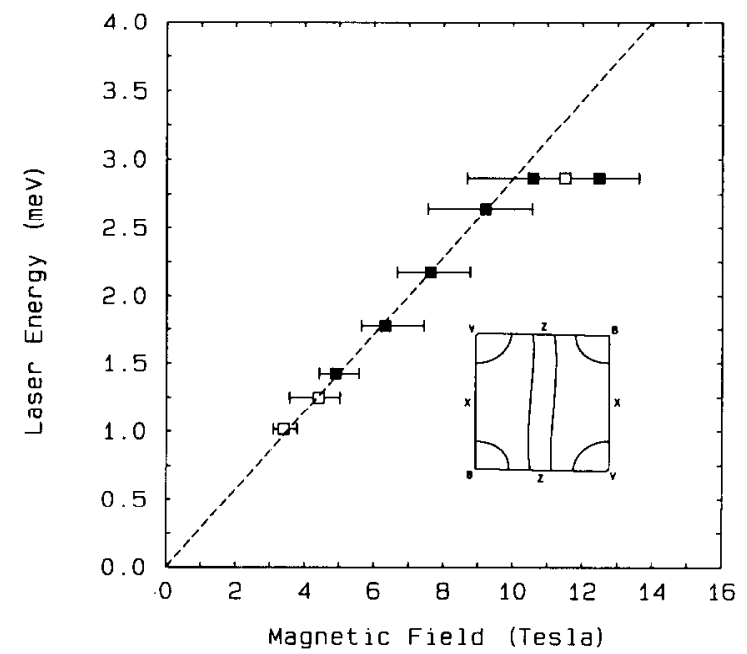

Fig. 1. Cyclotron resonance positions at each energy. Solid squares correspond to reflectance minima and open squares to maxima. The horizontal bars correspond to the estimated widths of the CR features. The dashed line corresponds to the fitted effective mass of $0.40 \mathrm{~m}_{\mathrm{e}}$. The inset shows the closed pocket in the calculated Fermi surface [12], which we associate with the primary $\mathrm{CR}$. bolometers were used to measure the incident and the reflected radiation, and the reflected signal was normalised to the incident signal. Magnetic fields were provided by a $20 \mathrm{~T}$ Bitter magnet.

The measured FIR magneto-reflectivity at $1.2 \mathrm{~K}$ is shown in fig. 2 for several different laser wavelengths. A number of features are visible in the traces, the strongest of which occurs at a field which is proportional to the FIR energy, the characteristic behaviour of CR. This feature appears as a dip in reflectivity at $5 \mathrm{~T}$ with $871 \mu \mathrm{m}$ radiation, and as such is very similar in form to $\mathrm{CR}$ reflectivity signals seen from $2 \mathrm{D}$ electron gases in semiconductor heterostructures when the underlying reflectivity of the structure is high [13]. In contrast to the $\sim 10 \%$ modulation of the reflectivity seen in the latter systems, however, the small skin-depth of $\mathrm{ET}_{2} \mathrm{KHg}(\mathrm{SCN})_{4}$ means that the CR leads to only a $-0.6 \%$ change in reflectivity. At higher energies the dip becomes wider and shallower, whereas for longer wavelengths the CR is observed as a peak. The latter behaviour is also seen in heterostructures under conditions of changing background reflectance [13]; the sharp change in behaviour between 871

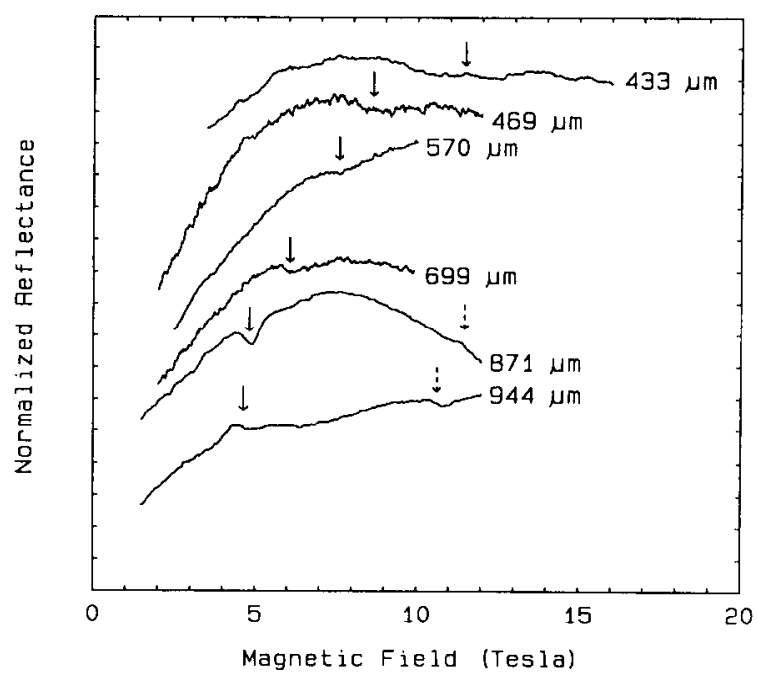

Fig. 2. Normalized magncto-reflectance of $\mathrm{ET}_{2} \mathrm{KHg}(\mathrm{SCN})_{4}$ at $1.2 \mathrm{~K}$ for different radiation energies. The primary series of $\mathrm{CR}$ features are marked with solid arrows and additional features with broken arrows. 
and $944 \mu \mathrm{m}$ may be due to a low energy lattice phonon. The field positions of the CR features are plotted against energy in fig. 1. An effective mass $m_{\text {cyc }}^{*}=(0.40 \pm 0.02) m_{\mathrm{e}}$ is estimated from the linear slope of the plot of the CR field position against the radiation energy; there is some evidence that either non-parabolicity or a resonant coupling mechanism [13] is leading to an effective mass which increases slightly with increasing energy. The corresponding increase in linewidth would seem to support the latter mechanism.

As seen in fig. 2, apart from the very clear CR corresponding to carriers with $m_{\text {cyc }}^{*} \sim 0.4 m_{\mathrm{e}}$, there is a weaker CR-like feature at higher fields with $m_{\text {cyc }}^{*} \sim(0.94 \pm 0.02) m_{\mathrm{e}}$. The significance of this feature is not clear at present; it may correspond to CR of carriers in another smaller pocket not observed in the transport measurements.

In order to measure the transport mass, MR measurements were performed at fields well below the kink transition at both ${ }^{3} \mathrm{He}$ and ${ }^{4} \mathrm{He}$ temperatures. Pairs of gold wires were attached on both sides of the platelets using platinum paint, and the longitudinal MR was measured using standard AC lock-in techniques; temperatures were measured using calibrated $\mathrm{RuO}$ and Ge resistance thermometers. The temperature dependence of the amplitude of the primary $670 \mathrm{~T}$ SdHo between 14 and $17 \mathrm{~T}$ is shown in fig. 3 ; it is consistent with an effective mass $m_{1 \mathrm{r}}^{*} \simeq$ $2.0 m_{\mathrm{e}}$, somewhat higher than the previously reported value of $1.4 m_{e}[10]$.

We must now relate $m_{\mathrm{tr}}$ to the effective masses seen in the CR measurements. The agreement between the MR data and the Hückel model prediction suggests that the only large closed section of Fermi surface is the 2D X-point hole pocket at the corner of the Brillouin zone. A closed Fermi surface is necessary for the observation of $C R$, and so, in the absence of any other detected or predicted closed pockets of carriers, we assign the strongest $\mathrm{DR}$ to the $2 \mathrm{D}$ carrier pocket observed in the high-field SdHo. Comparing data taken over comparable temperature ranges, the CR $(1.2 \mathrm{~K})$ yields an effective mass of $0.40 m_{\mathrm{e}}$ whilst the SdHo $(0.5-4.0 \mathrm{~K})$ give a value close to $2.0 \mathrm{~m}_{\mathrm{e}}$. This difference is probably

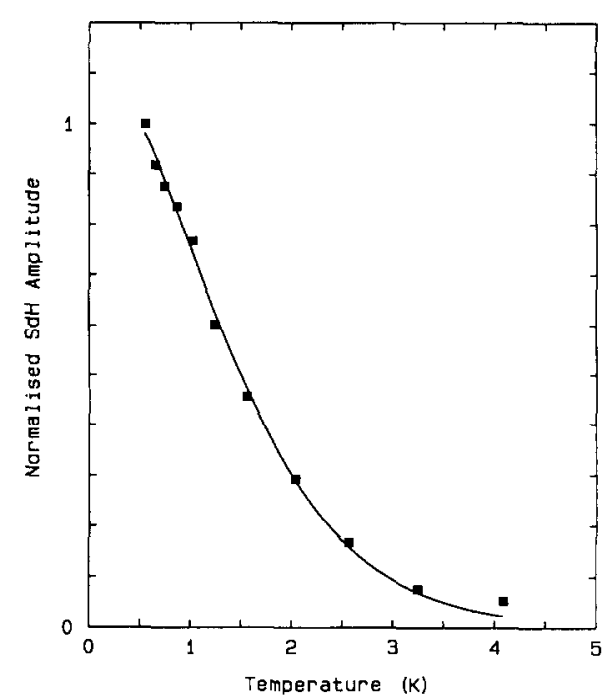

Fig. 3. Measured temperature dependence of the Fourier amplitude of the $670 \mathrm{~T}$ fundamental field Shubnikov-de Haas oscillations (solid squares) fitted using $m^{*} / m_{\mathrm{e}}=1.98$.

a consequence of Kohn's theorem [8, 14], which states that at long wavelengths an external perturbation only couples to centre-of-mass motion, which is independent of EEI. Thus CR, representing the excitation of a $q=0$ magnetoplasmon, measures the "bare" band-structure mass, renormalised only by electron-phonon interactions [13], whereas the transport mass, representing the $q=\infty$ case of a magnetoplasmon [14], is renormalised by EEI. Some violations of Kohn's theorem due to non-parabolicity have becn observed in CR in high mobility semiconductor heterojunctions $[14,15]$, but these tend to lead to a change in CR line shape, rather than a large shift in CR position. Thus a comparison between the measured $m_{\mathrm{cyc}}^{*}$ and $m_{\mathrm{tr}}^{*}$ for $\mathrm{ET}_{2} \mathrm{KHg}(\mathrm{SCN})_{4}$ represents a direct gauge of the strength of the EEI in a $2 \mathrm{D}$ system as measured by the renormalisation of the effective mass: in $\mathrm{ET}_{2} \mathrm{KHg}(\mathrm{SCN})_{4}$ the effective mass appears to be enhanced by a factor of $\sim 5$ by EEI at $1.2 \mathrm{~K}$. A similar effect may take place in the $11 \mathrm{~K}$ superconductor $\mathrm{ET}_{2} \mathrm{CU}(\mathrm{SCN})_{2}$, where the transport mass is $\sim 4$ times larger than that expected from band structure calculations [3].

Effective masses may also be derived from the 
band-structure calculations of Mori [12], yielding a cyclotron mass of $1.2 m_{\mathrm{e}}$ for the $\mathrm{X}$-point hole pocket. The corresponding calculated plasma frequencies are $0.77 \mathrm{eV}$ for $E$ polarised along $a$, and $0.35 \mathrm{eV}$ for $E$ along $c$. Experimental plasma frequencies in this material have been measured by Tamura et al. [17] to be $1.1 \mathrm{eV}$ with the $E$-vector polarised along $a$, and $0.63 \mathrm{eV}$ with the $E$-vector along $c$, suggesting that the true bandwidth is greater (and hence the effective mass is smaller) than predicted by Mori's calculations. Since the effective mass is inversely proportional to the square of the plasma frequency, we would expect a cyclotron mass of $0.47 \mathrm{~m}_{\mathrm{e}}$ on the basis of the measured plasma frequencies. This value is similar to the effective mass derived from the cyclotron resonance measurements. Thus both optical experiments, which are insensitive to electron-electron interactions, indicate a larger bandwidth than that predicted from band-structure calculations, which is in turn bigger than that deduced from transport measurements.

Several points remain for further study. The origin of the broadening of the CR as the magnetic field is increased is not clear. However, the field around which the CR broadens dramatically corresponds to a crossover in intensity between the two low-field SdHo series; the $800 \mathrm{~T}$ series begins to diminish in intensity at this field. Mechanisms for this transport phenomenon are discussed elsewhere [11]. There is also another weaker CR-like feature, and in this respect the behaviour of the $\mathrm{ET}_{2} \mathrm{KHg}(\mathrm{SCN})_{4}$ is similar to $\mathrm{TMTSF}_{2} \mathrm{ClO}_{4}$, where, in spite of the apparently simple quasi-one-dimensional bands, two CR are observed [7]. In such systems, the magnetic field induces an anisotropic 2D electron gas above a certain threshold field [17], and the observation of the CR in the TMTSF salt may be due to this mechanism. $\mathrm{ET}_{2} \mathrm{KHg}(\mathrm{SCN})_{4}$ also contains a $1 \mathrm{D}$ band which may be prone to anisotropic $2 \mathrm{D}$ electron gas formation in high magnetic fields, although no clear evidence of the consequent field-induced spin-density wave phases has been seen in ET salts. It should be noted that we have independently measured electron spin resonance (ESR) corresponding to $g^{*}=2$ in $\mathrm{ET}_{2} \mathrm{KHg}(\mathrm{SCN})_{4}$, and the subsidiary resonance is neither narrow enough, nor at a high enough field to be ESR.

In summary, we have measured cyclotron resonance in $\mathrm{ET}_{2} \mathrm{KHg}(\mathrm{SCN})_{4}$ for the first time; the results indicate a low carrier effective mass of $0.4 m_{\mathrm{e}}$. In contrast, transport measurements reveal a much higher effective mass $\sim 2.0 m_{\mathrm{e}}$. The difference between these two values is probably an indication of the strength of electron-electron interactions in the material.

\section{Acknowledgements}

This work is supported by the Science and Engineering Research Council in the UK, by Stichting voor Fundamenteel Onderzoek der Materie (FOM) in the Netherlands, and by the European Community Large Installations Plan.

\section{References}

[1] ET is BEDT-TTF or bis(ethylenedithio)-tetrathiafulvalene.

[2] T. Ishiguro and K. Yamaji, Organic Superconductors, Springer Series in Solid State Sciences, Vol. 88 (Springer, Berlin, 1990).

[3] F.L. Pratt, J. Singleton, M. Kurmoo, S.J.R.M. Spermon, W. Hayes and P. Day, Springer Proceedings in Physics 51 (1990) 200.

[4] N. Toyota, E.W. Fenton, T. Sasaki and M. Tachiki, Solid State Commun. 72 (1989) 859.

[5] M. Tokumoto, A.G. Swanson, J.S. Brooks, C.C. Agosta, S.T. Hannahs, N. Kinoshita, H. Anzai, M. Tamura, H. Tajima, H. Kuroda and J.R. Anderson, Proc. Int. Conf. on Organic Superconductors, Lake Tahoe (Plenum Press) to be published.

[6] I.D. Parker, D.D. Pigram, R.H. Friend, M. Kurmoo and P. Day, Synth. Metals 27 (1988) A387.

A.G. Swanson, J.S. Brooks, H. Ansai, N. Konoshita, M. Tokumoto and K. Murata, Solid State Commun. 73 (1990) 353

M. Tokumoto, A.G. Swanson, J.S. Brooks, M. Ta! mura, H. Tajima and H. Kuroda, Solid State Commun. 75 (1990) 439.

[7] A.S. Perel, J.S. Brooks, C.J.G.M. I angerak, T.J.B.M. Janssen, J. Singleton, J.A.A.J. Perenboom, G. Montambaux and L.Y. Chiang, preprint (1991).

[8] W. Kohn, Phys. Rev. 123 (1961) 1242.

[9] J. Larsen and C. Lenoir, Synthesis 134 (1988).

[10] T. Sasaki, N. Toyota, M. Tokumoto, N. Kinoshita and H. Anzai, Solid State Commun. 75 (1990) 97. 
T. Sasaki, H. Sato and N. Toyota, Synth. Metals 42 (1991) 2211.

T. Osada, R. Yagi, A. Kawasumi, S. Kagushima, N. Miura, M. Oshima, H. Mori, T. Nakamura and G. Saitoh, Synth. Metals 42 (1991) 2171.

[11] M. Doporto, F.L. Pratt, J. Singleton, T.J.B.M. Janssen, J.A.A.J. Perenboom, M. Kurmoo, W. Hayes and P. Day, Physica B 177 (1992) 333 (these Proceedings).

[12] H. Mori, S. Tanaka, M. Oshima, G. Saito, T. Mori, Y. Maruyama and H. Inokuchi, Bull. Chem. Soc. Jpn. 63 (1990) 2183.

[13] C.J.G.M. Langerak, J. Singleton, P.J. van der Wel, J.A.A.J. Perenboom, D.J. Barnes, R.J. Nicholas, M.A. Hopkins and C.T.B. Foxon, Phys. Rev. B 38 (1988) 13133.
[14] R.J. Nicholas, D.J. Barnes, R.G. Clark, S.R. Haynes, J.R. Mallett, A.M. Suckling, A. Usher, J.J. Harris, C.T. Foxon and R. Willett, Springer Series in SolidState Sciences, 87 (1989) 449.

[15] M. Watts, I. Auer, R.J. Nicholas, J.J. Harris and C.T. Foxon, Proc. Int. Conf. on the Applications of High Magnetic Fields in Semiconductor Physics, Würzburg (Springer) to be published.

[16] M. Tamura, R. Masuda, T. Naito, H. Tajima, H. Kuroda, A. Kobayashi, K. Yakushi, R. Kato, H. Kobayashi, M. Tokumoto, N. Kinoshita and H. Anzai, Synth. Metals 42 (1991) 2499.

[17] G. Montambaux and D. Poilblanc, Phys. Rev. B 37 (1988) 1913. 